Open J. Math. Sci., Vol. 2(2018),No.1, pp. 122 - 133

Website: https://pisrt.org/psr-press/journals/oms/

ISSN: 2523-0212 (Online) 2616-4906 (Print)

http://dx.doi.org/10.30538/oms2018.0022

\title{
ON MODULI AND CONSTANTS OF QUASI-BANACH SPACE
}

\author{
HUSSAIN MINHAJ UDDIN AHMAD QADRI ${ }^{1}$, QAISAR MEHMOOD
}

\begin{abstract}
In this article we discuss moduli and constants of quasi-Banach space and give some important properties of these moduli and constants. Moreover, we establish relationships of these moduli and constants with each other.
\end{abstract}

Mathematics Subject Classification: 46B20, 46E30.

Key words and phrases: von Neumann-Jordan Constant; James Constant; Modulus of uniform convexity; Smoothness; Uniform smoothness; Modulus of uniform smoothness; Non-squareness.

\section{Introduction}

The study on Banach space geometry provides many fundamental notions and interesting aspects, and sometimes has surprising results. The basic geometric properties such as convexity, smoothness, non-squareness have made great contributions to various fields of Banach space theory. Strict convexity of Banach spaces was first introduced in 1936 by Clarkson [1] as the property that the unit sphere contains no non-trivial line segments, that is, $1-\left\|2^{-1}(x+y)\right\|>0$ whenever $\|x\|=\|y\|=1$. Clarkson [1] made use of these values to define the uniform version of convexity to look at how convex the unit ball is in a space. And the modulus of convexity provides a quantification of the geometric structure of the space from the viewpoint of convexity. A situation similar to this also occurs in smoothness and other properties. A Banach space $\mathcal{B}$ is said to be smooth if each unit vector has a unique norm one support functional. In fact, this is equivalent to the statement that the norm is Gateaux differentiable, which happens iff $\lim _{t \rightarrow 0^{+}}=\sup (\|x+t y\|+\|x-t y\|) / 2-1=0$ where $x, y \in S_{\mathcal{B}}$ and

Received 15 September 2017. Revised 25 May 2018.

1 Corresponding Author

(C) 2018 Hussain Minhaj Uddin Ahmad Qadri, Qaisar Mehmood. This is an open access article distributed under the Creative Commons Attribution License, which permits unrestricted use, distribution, and reproduction in any medium, provided the original work is properly cited. 
$\|x\|=1$. This allows us to quantify the geometric structure of the space from the viewpoint of smoothness, namely, the modulus of smoothness of a Banach space $\mathcal{B}$. An advantage of these quantifications is that the complete duality between uniform convexity and uniform smoothness can be easily deduced by the well-known Lindenstrauss formulas, that is, a Banach space $\mathcal{B}$ is uniformly convex if and only if its dual space $\mathcal{B}^{*}$ is uniformly smooth. The same statement still holds if $\mathcal{B}$ is replaced with $\mathcal{B}^{*}$. Thus quantifying geometric structures might lead better results. Note that the same duality does not hold between strict convexity and smoothness in general, though one of those two properties of $\mathcal{B}^{*}$ implies the other of $\mathcal{B}$. There are some other ideas to quantify geometric structures of Banach spaces.

Many of the geometric constants for Banach Spaces have been investigated so far. These constants play an important role in the description of various geometric structures of Banach Spaces. In 1901 Jung [2] was the first who introduced a geometric constant for Banach Space. In 1936 and 1937 Clarkson [1, 3] introduced classical modulus of convexity to define a uniformly convex space. A great number of such moduli have been defined and introduced since then. The theory of the geometry of Banach Space has evolved very rapidly over the past fifty years. By contrast the study of quasi-Banach Space has lagged far behind, even though the first research papers in the subject appeared in the early 1940's [4].

There are very sound reasons to develop the understanding of these Space, but the absence of one of the fundamental tools of functional analysis, the HahnBanach theorem, has proved a very significant stumbling block. However, there has been some progress in the non-convex theory and arguably it has contributed to our appreciation of Banach space theory. A systematic study of quasi-Banach Space only really started in the late 1950's and early 1960's with the work of Klee, Peck, Rolewicz, Waelbroeck and Zelazko. The efforts of these researchers tended to go in rather separate directions. The subject was given great impetus by the paper of Duren, Romberg and Shields in 1969 which demonstrated both the possibilities for using quasi-Banach Space in classical function theory and also high-lighted some key problems related to the HahnBanach theorem. This opened up many new directions of research. The 1970's and 1980's saw a significant increase in activity with a number of authors contributing to the development of a coherent theory. An important breakthrough was the work of Roberts in [5] and [6], who showed that the Krein-Milman theorem fails in general quasi-Banach Space by developing powerful new techniques.

Quasi-Banach Space (Hp-Space when $p<1$ ) were also used significantly in Alexandrov's solution of the inner function problem in 1982 [7]. During this period three books on the subject appeared by Turpin [8], Rolewicz [9] (actually an expanded version of a book [6]. In the 1990's it seems to the author that while more and more analysts find that quasi-Banach Space have uses in their research, paradoxically the interest in developing a general theory has subsided somewhat. The strictly convex Banach spaces were introduced in 1936 by 
Clarkson, [1], who also studied the concept of uniform convexity. The uniform convexity of $L^{p}$ spaces, $1<p<\infty$, was also established in [1]. The concept of duality map was introduced by Beurling and Livingston [10] and was further developed by many others and, De Figueiredo [11]. General properties of the duality map can be found in De Figueiredo [11]. The most recent research work in this field is carried out by [12] and [13].

\section{Quasi-Banach Spaces}

This section collects some basic facts about quasi-Banach spaces. We restrict ourselves to [11] and the references therein for an extensive overview. If $\mathcal{B}$ is a (real) vector space, we say that $\|\cdot\|: \mathcal{B} \rightarrow[0, \infty)$ is a quasi-norm if

(1) $\|x\|=0$ if, and only if $x=0$

(2) $\|\alpha x\|=|\alpha|\|x\|$ for all $\alpha \in \mathbb{R}$ and $x \in \mathcal{B}$

(3) there is a constant $C \geq 1$, such that $\|x+y\| \leq C(\|x\|+\|y\|)$ for all $x, y \in \mathcal{B}$.

The constant $C$ is often referred to as the modulus of concavity, of the quasinorm. A very basic and important result is the Aoki-Rolewicz theorem. This result can be interpreted as saying that of $0<p \leq 1$ is given by $C=2^{q / p-1}$ then there is a constant $B$ so that for any $x_{1}, x_{2}, \ldots, x_{n} \in \mathcal{B}$ we have

$$
\left\|\sum_{k=1}^{n} x_{k}\right\| \leq B\left(\sum_{k=1}^{n}\left\|x_{k}\right\|^{p}\right)^{1 / p} .
$$

It is then possible to replace $\|$.$\| by an equivalent p$-subadditive quai-norm $\| \mid$. || so that

$$
\||x+y|\| \leq\left(\||x|\|^{p}+\||y|\|^{p}\right)^{1 / p}
$$

The space $\mathcal{B}$ is said to be $p$-normable if (1) holds. We will say that $\mathcal{B}$ is $p$-normed if the quasi-norm on $\mathcal{B}$ is $p$-subadditive.

If a quasi-normed vector space $\mathcal{B}$ is complete with respect to the metric induced by $\|$.$\| , it is called a quasi-Banach space.$

\section{Preliminaries}

Definition 3.1. For a non-trivial quasi-Banach space $\mathcal{B}$, the modulus of convexity is a function $\delta_{\mathcal{B}}:(0,2] \longrightarrow[0,1]$ defined as

$$
\delta_{\mathcal{B}}(\epsilon)=\inf \left\{1-\frac{\left\|x_{1}+x_{2}\right\|}{2 C}: \epsilon \in[0,2] C \geq 1, x_{1}, x_{2} \in S_{\mathcal{B}} ; \frac{\left\|x_{1}-x_{2}\right\|}{C} \epsilon\right\} .
$$

A characteristic or related coefficient of this modulus is:

$$
\delta_{0}(\mathcal{B})=\sup \left\{\epsilon \in[0,2]: \delta_{\mathcal{B}}(\epsilon)=0\right\} .
$$


Definition 3.2. Let $\mathcal{B}$ be a nontrivial quasi-Banach space and $S_{\mathcal{B}}$ be a unit ball in $\mathcal{B}$, the modulus of smoothness is a function $\rho_{\mathcal{B}}:[0, \infty) \longrightarrow[0, \infty)$ defined as,

$$
\begin{gathered}
\rho_{\mathcal{B}}(t)=\sup \left\{1-\frac{\left\|x_{1}+x_{2}\right\|}{2 C}: \forall x_{1}, x_{2} \in S_{\mathcal{B}} ; C \geq 1, \frac{\left\|x_{1}-x_{2}\right\|}{C} \leq t ; t \geq 0\right\}, \\
\rho_{1 \mathcal{B}}(t)=\sup \left\{\frac{\left\|x_{1}+t x_{2}\right\|+\left\|x_{1}-t x_{2}\right\|}{2 C}-\frac{1}{C}: x_{1}, x_{2} \in S_{\mathcal{B}} ; t \geq 0\right\}, \\
\rho_{2 \mathcal{B}}(t)=\sup \left\{\frac{1}{C}\left[\min \left(\left\|x_{1}+x_{2}\right\|,\left\|x_{1}-x_{2}\right\|\right)-1\right]: x_{1}, x_{2} \in S_{\mathcal{B}} ; t \geq 0\right\} .
\end{gathered}
$$

A characteristic or related coefficient of this modulus is

$$
\rho_{0}(\mathcal{B})=\lim _{t \rightarrow 0^{+}}\left(\frac{\rho_{\mathcal{B}}(t)}{t}\right)=\lim _{t \rightarrow 0^{+}}\left(\frac{\rho_{1 \mathcal{B}}(t)}{t}\right)=\lim _{t \rightarrow 0^{+}}\left(\frac{\rho_{2 \mathcal{B}}(t)}{t}\right) .
$$

Definition 3.3. For a non-trivial quasi-Banach space $\mathcal{B}$ the Yang-Wang Modulus is a function $\gamma_{\mathcal{B}}:[0,1] \longrightarrow[0,4]$ defined as,

$$
\gamma_{\mathcal{B}}(t)=\sup \left\{\frac{\left\|x_{1}+t x_{2}\right\|^{2}+\left\|x_{1}-t x_{2}\right\|^{2}}{2 C^{2}}: x_{1}, x_{2} \in S_{\mathcal{B}} ; t \in[0,1] ; C \geq 1\right\} \text {. }
$$

A characteristic or related coefficient of this modulus is,

$$
\gamma_{0}(\mathcal{B})=\lim _{t \rightarrow 0^{+}}\left(\frac{\gamma_{\mathcal{B}}(t)-\frac{1}{C}}{t}\right) .
$$

Definition 3.4. For a non-trivial quasi-Banach space $\mathcal{B}$, the von NeumannJordan constant is denoted by $C_{N J}(\mathcal{B})$ and is defined as the smallest constant $K$, for which the following estimate holds;

$$
\frac{1}{K} \leq \frac{\left\|x_{1}+x_{2}\right\|^{2}+\left\|x_{1}-x_{2}\right\|^{2}}{2 C^{2}\left(\left\|x_{1}\right\|^{2}+\left\|x_{2}\right\|^{2}\right)} \leq K
$$

where $x_{1}, x_{2} \in S_{\mathcal{B}}$ with $\left(x_{1}, x_{2}\right) \neq(0,0)$ and $C \geq 1$.

Equivalently, it can be written as,

$$
C_{N J}(\mathcal{B})=\sup \left\{\frac{\left\|x_{1}+x_{2}\right\|^{2}+\left\|x_{1}-x_{2}\right\|^{2}}{2 C^{2}\left(\left\|x_{1}\right\|^{2}+\left\|x_{2}\right\|^{2}\right)}: x_{1}, x_{2} \in S_{\mathcal{B}} ; C \geq 1\right\} .
$$

The parametrized form of this constant is,

$$
C_{N J}(\mathcal{B})=\sup \left\{\frac{\left\|x_{1}+t x_{2}\right\|^{2}+\left\|x_{1}-t x_{2}\right\|^{2}}{2 C^{2}\left(1+t^{2}\right)} ; t \in[0,1] ; C \geq 1\right\},
$$


where $S_{\mathcal{B}}$ is unit sphere and $x_{1}, x_{2} \in S_{\mathcal{B}}$. By taking $t=1$ and $x_{1}=x_{2}=x$, we obtain

$$
C_{N J}(\mathcal{B})=\frac{\|2 x\|^{2}}{2 C^{2}(1+1)} \geq \frac{2^{2}}{2 C(1+1)}=\frac{1}{C} .
$$

Definition 3.5. For a non-trivial quasi-Banach space $\mathcal{B}$ the James Gao-Lau constant is defined as

$$
J(\mathcal{B})=\sup \left\{\frac{1}{C} \min \left(\left\|x_{1}+x_{2}\right\|,\left\|x_{1}-x_{2}\right\|\right): x_{1}, x_{2} \in S_{\mathcal{B}} ; C \geq 1\right\} .
$$

The parametrized form of this constant is,

$$
J(\mathcal{B})=\sup \left\{\frac{1}{C} \min \left(\left\|x_{1}+t x_{2}\right\|,\left\|x_{1}-t x_{2}\right\|\right): C \geq 1 ; t \in[0,1]\right\} .
$$

\section{Main Results}

Here are some basic algebraic inequalities. These inequalities are very important. With the help of these inequalities, we will be able to proceed some main results.

Lemma 4.1. Every convex function $f$ with convex domain in $\mathbb{R}$ is continuous.

Lemma 4.2. [12] For all $0 \leq a \leq 2$ and $0 \leq b \leq 2$ we have

$$
\frac{a^{2}+b^{2}}{4 c^{2}} \leq\left(\frac{a+b}{2 c}-\frac{1}{c}\right)^{2}+\frac{1}{c^{2}}
$$

Lemma 4.3. [12] For all $a \in \mathbb{R}, b>0$ and $t \geq 0$ we have

$$
\frac{a t^{2}+b^{2}}{1+t^{2}} \leq \frac{a+\sqrt{a^{2}+b^{2}}}{2} .
$$

Lemma 4.4. [12] Let $1<p<\infty$ and $0 \leq t \leq 1$, then the function;

$$
\phi(t)=(1+t)^{p} /\left(1+t^{p}\right),
$$

is strictly increasing.

Lemma 4.5. [12] Let $0<r \leq 1$ and $0 \leq t \leq 1$, then $\forall s, t \in[0, \infty)$

$$
\left|t^{r}-s^{r}\right| \leq|t-s|^{r}
$$

Lemma 4.6. Let $0 \leq a \leq 1$ and $r_{0}=\frac{\sqrt{4+(1+a)^{4}}-(1+a)^{2}}{2}$, then

(1) $a \leq r_{0} \quad$ if $\quad a \in[0, \sqrt{2}-1]$

(2) $a \geq r_{0} \quad$ if $\quad a \in[\sqrt{2}-1,1]$

Proof. Let $a \in[0, \sqrt{2}-1]$, then

$$
\begin{aligned}
4+(1+a)^{4}-\left[(1+a)^{2}+2 a\right]^{2} & =4\left(1-a-3 a^{2}-a^{3}\right) \\
& =-4(a+1)(a+1+\sqrt{2})(a+1-\sqrt{2}) \\
& \geq 0 .
\end{aligned}
$$


This implies that

$$
\begin{array}{r}
\sqrt{4+(1+a)^{4}} \geq\left[(1+a)^{2}+2 a\right] \\
r_{0} \geq a .
\end{array}
$$

Let $a \in[\sqrt{2}-1,1]$, then we can prove it similarly.

Lemma 4.7. Let $\mathcal{B}$ be a quasi-Banach space, then for every $x \in \mathcal{B}$ with $\|x\|=1$ and $x^{*} \in X^{*}$ with $\left\|x^{*}\right\|=1$

$$
\begin{aligned}
& \rho_{\mathcal{B}}(\tau)=\sup \left\{\frac{\tau \epsilon}{2}-\delta_{\mathcal{B}^{*}}(\epsilon): \tau>0 ; \epsilon \in(0,2]\right\}, \\
& \rho_{\mathcal{B}^{*}}(\tau)=\sup \left\{\frac{\tau \epsilon}{2}-\delta_{\mathcal{B}}(\epsilon): \tau>0 ; \epsilon \in(0,2]\right\} .
\end{aligned}
$$

Proof. Let $\tau>0$ and $\epsilon \in(0,2] \& x, y \in S_{\mathcal{B}}$ with $\|x\|=1,\|y\|=1$. Then by Hahn-Banach theorem, $\exists x^{*}, y^{*} \in S_{\mathcal{B}}^{*}$ with $\left\|x^{*}\right\|=1,\left\|y^{*}\right\|=1$ such that;

Then,

$$
\begin{aligned}
\|x+y\| & =\left\langle x+y, x_{0}^{*}\right\rangle \\
\|x-y\| & =\left\langle x-y, y_{0}^{*}\right\rangle .
\end{aligned}
$$

$$
\begin{aligned}
\|x+y\|+\tau\|x-y\|-2 C & =\left\langle x+y, y_{0}^{*}\right\rangle+\tau\left\langle x-y, x_{0}^{*}\right\rangle-2 C \\
& =\left\langle x, x_{0}^{*}+\tau y_{0}^{*}\right\rangle+\left\langle y, x_{0}^{*}-\tau y_{0}^{*}\right\rangle-2 C \\
& =\left\|x_{0}^{*}+\tau y_{0}^{*}\right\|+\left\|x_{0}^{*}-\tau y_{0}^{*}\right\|-2 C .
\end{aligned}
$$

Therefore, we get

$$
\frac{\|x+y\|+\tau\|x-y\|}{2 C}-1=\frac{\left\|x_{0}^{*}+\tau y_{0}^{*}\right\|+\left\|x_{0}^{*}-\tau y_{0}^{*}\right\|}{2 C}-1 .
$$

Now, $\|x-y\|<\epsilon$ so, we get

$$
\frac{\|x+y\|}{2 C}-1+\frac{\tau \epsilon C}{2 C}=\frac{\left\|x_{0}^{*}+\tau y_{0}^{*}\right\|+\left\|x_{0}^{*}-\tau y_{0}^{*}\right\|}{2 C}-1 .
$$

Taking supremum on both sides, with $\|x\|=\|y\|=1$ and $\left\|x^{*}\right\|=\left\|y^{*}\right\|=1$ we have,

$$
\begin{gathered}
\sup \left\{\frac{\|x+y\|}{2 C}-1\right\}+\frac{\tau \epsilon C}{2 C}=\sup \left\{\frac{\left\|x_{0}^{*}+\tau y_{0}^{*}\right\|+\left\|x_{0}^{*}-\tau y_{0}^{*}\right\|}{2 C}-1\right\} \\
-\delta_{\mathcal{B}}(\epsilon)+\frac{\tau \epsilon}{2}=\rho_{\mathcal{B}^{*}}(\tau) \\
\rho_{\mathcal{B}^{*}}(\tau)=\frac{\tau \epsilon}{2}-\delta_{\mathcal{B}}(\epsilon) . \\
\rho_{\mathcal{B}^{*}}(\tau) \geq \sup \left\{\frac{t \epsilon}{2}-\delta_{\mathcal{B}}(\epsilon): t>0 ; \epsilon \in(0,2]\right\} .
\end{gathered}
$$


Now to prove the reverse inequality, we proceed as:

Let $x^{*}, y^{*} \in S_{\mathcal{B}}^{*}$ with $\left\|x^{*}\right\|=1,\left\|y^{*}\right\|=1$ and let $\delta>0$ then for every $\tau>0$ $\exists x, y \in S_{\mathcal{B}}$ with $\|x\|=1,\|y\|=1$ such that;

$$
\begin{gathered}
\left\|x^{*}+\tau y^{*}\right\|=\left\langle x_{0}+\tau y^{*}, x^{*}\right\rangle+\epsilon \\
\left\|x^{*}-\tau y^{*}\right\|=\left\langle y_{0} \tau y^{*}, x^{*}\right\rangle+\epsilon .
\end{gathered}
$$

Then,

$$
\begin{aligned}
\left\|x^{*}+\tau y^{*}\right\|+\left\|x^{*}-\tau y^{*}\right\|-2 C & =\left\langle x_{0}, x^{*}+\tau y^{*}\right\rangle+\left\langle y_{0}, x^{*}-\tau y^{*}\right\rangle-2 C+2 \epsilon \\
& =\left\langle x_{0}+y_{0}, x^{*}\right\rangle+\tau\left\langle x_{0}-y_{0}, y^{*}\right\rangle-2 C+2 \epsilon \\
& =\left\|x_{0}+y_{0}\right\|+\tau\left\|x_{0}-y_{0}\right\|-2 C+2 \epsilon \\
& =\left\|x_{0}+y_{0}\right\|-2 C+\tau\left\|x_{0}-y_{0}\right\|+2 \epsilon .
\end{aligned}
$$

Now since, $\|x-y\| \leq \epsilon$ so, we get

$$
\begin{aligned}
\frac{\left\|x^{*}+\tau y^{*}\right\|+\left\|x^{*}-\tau y^{*}\right\|-2 C}{2 C} & =\frac{\left\|x_{0}+y_{0}\right\|-2 C+\tau \epsilon C}{2 C}++\frac{\epsilon}{C} \\
& =\frac{\left\|x_{0}+y_{0}\right\|-2 C}{2 C}+\frac{\tau \epsilon C}{2 C}+\frac{\epsilon}{C} \\
& =\frac{\left\|x_{0}+y_{0}\right\|}{2 C}-1+\frac{\tau \epsilon}{2}++\frac{\epsilon}{C} .
\end{aligned}
$$

Taking supremum on both sides, with $\|x\|=\|y\|=1$ and $\left\|x^{*}\right\|=\left\|y^{*}\right\|=1$ we have,

$$
\begin{gathered}
\sup \left\{\frac{\left\|x^{*}+\tau y^{*}\right\|+\left\|x^{*}-\tau y^{*}\right\|}{2 C}-1\right\}=\sup \left\{\frac{\left\|x_{0}+y_{0}\right\|}{2 C}-1\right\}+\frac{\tau \epsilon}{2}+\frac{\epsilon}{C} \\
\rho_{\mathcal{B}^{*}}(\tau)=-\delta_{\mathcal{B}}(\epsilon)+\frac{\tau \epsilon}{2}+\frac{\epsilon}{C} \\
\rho_{\mathcal{B}^{*}}(\tau)=\frac{\tau \epsilon}{2}-\delta_{\mathcal{B}}(\epsilon)+\frac{\epsilon}{C} . \\
\rho_{\mathcal{B}^{*}}(\tau) \leq \sup \left\{\frac{\tau \epsilon}{2}-\delta_{\mathcal{B}}(\epsilon): \tau>0, \epsilon \in(0,2]\right\} .
\end{gathered}
$$

Hence, by combining (14) and (15) we get

$$
\rho_{\mathcal{B}^{*}}(\tau)=\sup \left\{\frac{\tau \epsilon}{2}-\delta_{\mathcal{B}}(\epsilon): \tau>0 ; \epsilon \in(0,2]\right\} \text {. }
$$

Lemma 4.8. Let $\mathcal{B}$ be quasi-Banach Space. $\forall 0 \leq \epsilon_{1} \leq J(\mathcal{B}) \leq 2$;

$$
\delta_{\mathcal{B}}(\epsilon) \geq \frac{2(1-J(\mathcal{B}))}{2-J(\mathcal{B})}+\left(\frac{J(\mathcal{B})}{2(2-J(\mathcal{B}))}\right) \epsilon .
$$


Proof. Since for all $0 \leq \epsilon \leq J(\mathcal{B}) \leq 2$, we have

$$
\frac{\delta_{\mathcal{B}}\left(J(\mathcal{B})-\delta_{\mathcal{B}}(\epsilon)\right.}{J(\mathcal{B})-\epsilon} \leq \frac{1-\delta_{\mathcal{B}}(\epsilon)}{2-\epsilon}
$$

(for simplicity we shall consider from now $\delta_{\mathcal{B}}=\delta$ and $J(\mathcal{B})=J$ )

$$
\begin{aligned}
(\delta(J)-\delta(\epsilon))(2-\epsilon) & \leq(1-\delta(\epsilon))(J-\epsilon) \\
2(\delta(J)-\delta(\epsilon))-\epsilon(\delta(J)-\delta(\epsilon)) & \leq(J(1-\delta(\epsilon))-\epsilon(1-\delta(\epsilon))) \\
2 \delta(J)-2(\delta(\epsilon))-\epsilon \delta(J) & \leq(J-J \delta(\epsilon))-\epsilon \\
\epsilon-\epsilon \delta(J)+2 \delta(J)-J & \leq 2 \delta(\epsilon)-J \delta(\epsilon) \\
\epsilon(1-\delta(J))+2 \delta(J)-J & \leq(2-J) \delta(\epsilon) .
\end{aligned}
$$

Since we know that $1-\frac{J}{2} \leq \delta(J)$ this implies $1-\delta(J) \leq \frac{J}{2}$. Therefore, we get

$$
\begin{aligned}
\epsilon\left(\frac{J}{2}\right)+2(\delta(J))-J & \leq(2-J) \delta(\epsilon) \\
\frac{J \epsilon+4-2 J-2 J}{2(2-J)} & \leq \delta(\epsilon) \\
\frac{J \epsilon+4-4 J}{2(2-J)} & \leq \delta(\epsilon) \\
\frac{J \epsilon}{2(2-J)}+\frac{4-4 J}{2(2-J)} & \leq \delta(\epsilon) .
\end{aligned}
$$

It can be re-arranged as

$$
\begin{aligned}
\delta(\epsilon) & \geq\left(\frac{J}{2(2-J)}\right) \epsilon+\frac{4-4 J}{2(2-J)} \\
\delta(\epsilon) & \geq \frac{4-4 J}{2(2-J)}+\left(\frac{J}{2(2-J)}\right) \epsilon \\
\left.\delta_{(} \epsilon\right) & \geq \frac{2(1-J)}{2-J}+\left(\frac{J}{2(2-J)}\right) \epsilon \\
\delta_{\mathcal{B}}(\epsilon) & \geq \frac{2(1-J(\mathcal{B}))}{2-J(\mathcal{B})}+\left(\frac{J(\mathcal{B})}{2(2-J(\mathcal{B}))}\right) \epsilon .
\end{aligned}
$$

Lemma 4.9. Let $\mathcal{B}$ be a quasi-Banach Space. For all $0 \leq J(\mathcal{B}) \leq \epsilon \leq 2$

$$
\delta_{\mathcal{B}}(\epsilon) \geq\left[\frac{1}{J(\mathcal{B})}-\frac{1}{2}\right] \epsilon .
$$

Proof. Since we know that $\frac{\delta(\epsilon)}{\epsilon}$ is a non-decreasing function, therefore we can have,

$$
\frac{\delta(J)}{J} \leq \frac{\delta(\epsilon)}{\epsilon}
$$


This can be written as,

$$
\begin{aligned}
& \frac{\delta(\epsilon)}{\epsilon} \geq \frac{\delta(J)}{J} \\
& \delta(\epsilon) \geq\left(\frac{\delta(J)}{J}\right) \epsilon .
\end{aligned}
$$

But, we know that

$$
1-\frac{J}{2} \leq \delta(J)
$$

Therefore, we have;

$$
\begin{aligned}
\delta(\epsilon) & \geq\left(\frac{1-\frac{J}{2}}{J}\right) \epsilon \\
& =\left(\frac{2-J}{2 J}\right) \epsilon \\
\delta(\epsilon) & \geq\left[\frac{1}{J}-\frac{1}{2}\right] \epsilon \\
\delta_{\mathcal{B}}(\epsilon) & \geq\left[\frac{1}{J(\mathcal{B})}-\frac{1}{2}\right] \epsilon .
\end{aligned}
$$

Hence Proof

Theorem 4.10. Let $\mathcal{B}$ be a quasi-Banach Space. Then

$$
\rho_{\mathcal{B}}(1) \leq 2\left(1-\frac{1}{J(\mathcal{B})}\right) .
$$

Proof. If $J(\mathcal{B})=2$ or if $\mathcal{B}$ is not uniformly non-square, then $\rho_{\mathcal{B}}(1)=1$ and there is nothing to prove.

Assume $J(\mathcal{B})<2$. According to Lemma (4.7), we have

$$
\rho_{\mathcal{B}}(1)=\sup \left\{\frac{\epsilon}{2}-\delta_{\mathcal{B}}^{*}(\epsilon)\right\} .
$$

Here we just need to show that

$$
\frac{\epsilon}{2}-\delta_{\mathcal{B}}^{*}(\epsilon) \leq 2\left(1-\frac{1}{J(\mathcal{B})}\right) .
$$

Let $0<\epsilon \leq J$ and if $\epsilon \leq 4\left(1-\frac{1}{J}\right)$ then the equality in (20) occurs.

Case 1. Let $4\left(1-\frac{1}{J}\right)<\epsilon \leq J$ then by Lemma 4.8 , we have

$$
\delta_{\mathcal{B}}^{*}(\epsilon) \geq \frac{2(1-J)}{2-J}+\left(\frac{J}{2(2-J)}\right) \epsilon .
$$

From which it follows that

$$
\delta_{\mathcal{B}}(\epsilon) \geq \frac{2-2 J}{2-J}+\left(\frac{J}{2(2-J)}\right) \epsilon,
$$


subtracting from $\frac{\epsilon}{2}$ on both sides

$$
\begin{aligned}
\frac{\epsilon}{2}-\delta_{\mathcal{B}}(\epsilon) & \geq \frac{\epsilon}{2}-\left[\frac{2-2 J}{2-J}+\left(\frac{J}{2(2-J)}\right) \epsilon\right] \\
& =\left(\frac{1-J}{2-J}\right) \epsilon-\frac{2-2 J}{2(2-J)} \\
& \leq\left(\frac{1-J}{2-J}\right) 4\left(1-\frac{1}{J}\right)-\frac{2-2 J}{2(2-J)} \\
& \leq 2\left(1-\frac{1}{J}\right) .
\end{aligned}
$$

Case 2. Let $J<\epsilon \leq 2$ then by Lemma 4.9 ,

$$
\begin{aligned}
\delta_{\mathcal{B}}(\epsilon) & \geq\left(\frac{1}{J}-\frac{1}{2}\right) \epsilon \\
\frac{\epsilon}{2}-\delta_{\mathcal{B}}(\epsilon) & \geq \frac{\epsilon}{2}-\left(\frac{1}{J}-\frac{1}{2}\right) \epsilon \\
& =\left(1-\frac{1}{J}\right) \epsilon \leq 2\left(1-\frac{1}{J}\right) .
\end{aligned}
$$

This completes the proof.

Theorem 4.11. Let $\mathcal{B}$ be a quasi-Banach Space. Then

$$
C_{N J}(\mathcal{B}) \leq 1+C \rho_{\mathcal{B}}(1)\left[\sqrt{\left(1-C \rho_{\mathcal{B}}(1)\right)^{2}+1}-\left(1-C \rho_{\mathcal{B}}(1)\right)\right]
$$

Proof. The proof can be consulted from [12, Theorem 3.3]

Corollary 4.12. Let $\mathcal{B}$ be a quasi-Banach Space such that,

$$
\rho_{\mathcal{B}}(1) \leq \sqrt{J(\mathcal{B})-1}
$$

Then, the equality holds in (23) if $\mathcal{B}$ is not uniformly non-square.

Proof. By Lemma 4.10, we have

$$
\rho_{\mathcal{B}}(1) \leq 2\left(1-\frac{1}{J}\right) \leq \sqrt{J(\mathcal{B})-1}
$$

If $\mathcal{B}$ is not uniformly non-square then the equality holds in (24). If the equality holds in (19) then we have the equality in the second inequality of (24) which shows that either $J(\mathcal{B})=2$ or $\mathcal{B}$ is uniformly non-square. This completes the proof.

Corollary 4.13. Let $\mathcal{B}$ be a quasi-Banach Space, then

$$
\sqrt{J(\mathcal{B})-1} \leq \frac{J(\mathcal{B})}{2}
$$


Proof. Let

$$
\sqrt{J(\mathcal{B})-1} \leq \frac{J(\mathcal{B})}{2}
$$

Taking square on both sides

$$
J(\mathcal{B})-1 \leq\left[\frac{J(\mathcal{B})}{2}\right]^{2} .
$$

This can be written as

$$
\begin{gathered}
{\left[\frac{J(\mathcal{B})}{2}\right]^{2} \geq J(\mathcal{B})-1 .} \\
J(\mathcal{B})^{2} \geq 4 J(\mathcal{B})-4 . \\
(J(\mathcal{B})-2)^{2} \geq 0 .
\end{gathered}
$$

This completes the proof.

Corollary 4.14. For a quasi-Banach space $\mathcal{B}$ the inequality

$$
C_{N J}(\mathcal{B}) \leq J(\mathcal{B})+\sqrt{J(\mathcal{B})-1}+\left[\sqrt{1+(1-\sqrt{J(\mathcal{B})-1})^{2}}-1\right] .
$$

holds if and only if $\mathcal{B}$ is not uniformly non-square.

Proof. Let

$$
f(t)=1+t\left[\sqrt{(1+t)^{2}+1}-(1-t)\right] .
$$

The function $f(t)$ is strictly increasing and hence by Corollary 4.12,

$$
C_{N J}(\mathcal{B}) \leq f\left(\rho_{\mathcal{B}}(1)\right) \leq f(\sqrt{J(\mathcal{B})-1}),
$$

which implies (26).

We can immediately see that the equality in (26) holds if $\mathcal{B}$ is not uniformly non-square. If the equality in (26) holds then we have,

$$
f\left(\rho_{\mathcal{B}}(1)\right) \leq f(\sqrt{J(\mathcal{B})-1}) .
$$

Which shows that

$$
\rho_{\mathcal{B}}(1) \leq \sqrt{J(\mathcal{B})-1} .
$$

Which implies that $\mathcal{B}$ is not uniformly non-square by Corollary 4.12 . This completes the proof.

Corollary 4.15. Let $\mathcal{B}$ be a quasi-Banach Space. Then

$$
C_{N J}(\mathcal{B}) \leq J(\mathcal{B})+\sqrt{J(\mathcal{B})-1}\left[\sqrt{\left(1-C \rho_{\mathcal{B}}(1)\right)^{2}+1}-1\right] .
$$

Proof. The proof is obvious on the same pattern as we have done in the above corollaries.

Theorem 4.16. Let $\mathcal{B}$ be a non-trivial quasi-Banach space, then

$$
C_{N J}(\mathcal{B})=\sup \left\{\frac{\gamma_{\mathcal{B}}(t)}{1+t^{2}}: t \in[0,1]\right\} .
$$


Proof. Since we know that the parametric form of the von-Numan james constant is,

$$
\begin{aligned}
C_{N J}(\mathcal{B}) & =\sup \left\{\frac{\left\|x_{1}+t x_{2}\right\|^{2}+\left\|x_{1}-t x_{2}\right\|^{2}}{2 C^{2}\left(1+t^{2}\right)}: t \in[0,1] ; C \geq 1\right\} \\
& =\sup \left\{\frac{\gamma_{\mathcal{B}}(t)}{1+t^{2}}: t \in[0,1]\right\} .
\end{aligned}
$$

\section{Competing interests}

The authors declare that they have no competing interests.

\section{REFERENCES}

1. Clarkson, J. A. (1936). Uniformly convex spaces. Transactions of the American Mathematical Society, 40(3), 396-414. https://doi.org/10.1090/S0002-9947-1936-1501880-4

2. Jung, H. W. E. (1899). ber die kleinste kugel die eine rumliche figur einschliesst... Marburg..

3. Clarkson, J. A. (1937). The von Neumann-Jordan constant for the Lebesgue spaces. Ann. of Math., 38, 114-115.

4. Day, M. M. (1940). The spaces $L^{p}$ with $0<p<1$. Bulletin of the American Mathematical Society, 46(10), 816-823.

5. Roberts, J. W. (1975). Pathological compact convex sets in $L_{p}[0,1], 0 \leq p<1$. In The Altgeld Book, University of Illinois Functional Analysis Seminar (Vol. 1976).

6. Roberts, J. W. (1977). A nonlocally convex F-space with the Hahn-Banach approximation property. In Banach spaces of analytic functions (pp. 76-81). Springer, Berlin, Heidelberg.

7. Aleksandrov, A. B. (1983). The existence of inner functions in the ball. Mathematics of the USSR-Sbornik, 46(2), 143. https://doi.org/10.1070/SM1983v046n02ABEH002759

8. Turpin, P. Convexities in general topological vector spaces. Dissertationes Math

9. Rolewicz, S. (1957). On a certain class of linear metric spaces. Bull. Acad. Polon. Sci, 5, 471-473.

10. Beurling, A., \& Livingston, A. E. (1962). A theorem on duality mappings in Banach spaces. Arkiv fr Matematik, 4(5), 405-411.

11. de Figueiredo, D. G. (1967). Topics in Nonlinear Functional Analysis (Vol. 48), University of Maryland. Institute for Fluid Dynamics and Applied Mathematics.

12. Kwun, Y. C., Mehmood, Q., Nazeer, W., Haq, A. U., \& Kang, S. M. (2016). Relations between generalized von Neumann-Jordan and James constants for quasi-Banach spaces. Journal of Inequalities and Applications, 2016(1), 171. https://doi.org/10.1186/s13660016-1115-z

13. Nazeer, W., Mehmood, Q., Kang, S. M., \& Haq, A. U. (2018). Generalized von NeumannJordan and James Constants for Quasi-Banach Spaces. Journal of Computational Analysis and Applications, 25(6),1043-1052.

\section{Hussain Minhaj Uddin Ahmad Qadri}

Aitchison College, Lahore 54000, Pakistan.

e-mail: minhaj_h@hotmail.com

Qaisar Mehmood

Department of Mathematics, Govt. Science College, Wahdat Road, Lahore Pakistan.

e-mail: qaisar47@hotmail.com 